\section{Mutations of the BRAF gene in human cancer}

Helen Davies ${ }^{1,2}$, Graham R. Bignell ${ }^{1,2}$, Charles Cox ${ }^{1,2}$, Philip Stephens ${ }^{1,2}$, Sarah Edkins ${ }^{1}$, Sheila Clegg ${ }^{1}$, Jon Teague ${ }^{1}$, Hayley Woffendin ${ }^{1}$, Mathew J. Garnett ${ }^{3}$, William Bottomley ${ }^{1}$, Neil Davis ${ }^{1}$, Ed Dicks ${ }^{1}$, Rebecca Ewing ${ }^{1}$, Yvonne Floyd ${ }^{1}$, Kristian Gray ${ }^{1}$, Sarah Hall ${ }^{1}$, Rachel Hawes ${ }^{1}$, Jaime Hughes ${ }^{1}$, Vivian Kosmidou ${ }^{1}$, Andrew Menzies ${ }^{1}$, Catherine Mould ${ }^{1}$, Adrian Parker ${ }^{1}$, Claire Stevens ${ }^{1}$, Stephen Watt ${ }^{1}$, Steven Hooper ${ }^{3}$, Rebecca Wilson ${ }^{3}$, Hiran Jayatilake ${ }^{4}$, Barry A. Gusterson ${ }^{5}$, Colin Cooper ${ }^{6}$, Janet Shipley ${ }^{6}$, Darren Hargrave ${ }^{7}$, Katherine Pritchard-Jones $^{7}$, Norman Maitland ${ }^{8}$, Georgia Chenevix-Trench ${ }^{9}$, Gregory J. Riggins ${ }^{10}$, Darell D. Bigner ${ }^{10}$, Giuseppe Palmieri ${ }^{11}$, Antonio Cossu ${ }^{12}$, Adrienne Flanagan ${ }^{13}$, Andrew Nicholson ${ }^{14}$ Judy W. C. Ho ${ }^{15}$, Suet Y. Leung ${ }^{16}$, Siu T. Yuen ${ }^{16}$, Barbara L. Weber ${ }^{17}$, Hilliard F. Seigler ${ }^{18}$, Timothy L. Darrow ${ }^{18}$, Hugh Paterson ${ }^{3}$, Richard Marais $^{3}$, Christopher J. Marshall ${ }^{3}$, Richard Wooster ${ }^{1,6}$, Michael R. Stratton ${ }^{1,4}$ \& P. Andrew Futreal

${ }^{1}$ Cancer Genome Project, The Wellcome Trust Sanger Institute, Wellcome Trust Genome Campus, Hinxton, CB10 1SA, UK

${ }^{3}$ Cancer Research UK Centre for Cell and Molecular Biology, Chester Beatty Labs, Institute of Cancer Research, London SW3 6JB, UK

${ }^{4}$ Section of Cancer Genetics; ${ }^{6}$ Section of Molecular Carcinogenesis; and ${ }^{7}$ Section of Paediatrics, Institute of Cancer Research, Sutton, Surrey SM2 5NG, UK

${ }^{5}$ Department of Pathology, Western Infirmary, University of Glasgow, S11 6NT, UK

${ }^{8}$ Department of Biology, YCR Cancer Research Unit, University of York, York YO10 5YW, UK

${ }^{9}$ Queensland Institute of Medical Research, RBH Post Office Herston, Queensland 4029, Australia

${ }^{10}$ Department of Pathology, and ${ }^{18}$ Department of Surgery, Duke University Medical Centre, Durham, North Carolina 27710, USA

${ }^{11}$ Institute of Molecular Genetics, C.N.R., Loc. Tramariglio, Alghero 07040, Italy

${ }^{12}$ Department of Pathology, University of Sassari, Azienda USL1, Sassari 07100, Italy

${ }^{13}$ Royal Free \& University College Medical School, London WC1E 6JJ, UK

${ }^{14}$ Royal Brompton Hospital, London SW3 6NP, UK

${ }^{15}$ Department of Surgery, and ${ }^{16}$ Department of Pathology, The University of Hong Kong, Queen Mary Hospital, Hong Kong

${ }^{17}$ Abramson Family Cancer Research Institute, University of Pennsylvania Cancer Center, Philadelphia, Pennsylvania 19104, USA

${ }^{2}$ These authors contributed equally to this work

Cancers arise owing to the accumulation of mutations in critical genes that alter normal programmes of cell proliferation, differentiation and death. As the first stage of a systematic genomewide screen for these genes, we have prioritized for analysis signalling pathways in which at least one gene is mutated in human cancer. The RAS-RAF-MEK-ERK-MAP kinase pathway mediates cellular responses to growth signals ${ }^{1}$. RAS is mutated to an oncogenic form in about $15 \%$ of human cancer. The three $R A F$ genes code for cytoplasmic serine/threonine kinases that are regulated by binding $\mathrm{RAS}^{1-3}$. Here we report $B R A F$ somatic missense mutations in $66 \%$ of malignant melanomas and at lower frequency in a wide range of human cancers. All mutations are within the kinase domain, with a single substitution (V599E) accounting for $80 \%$. Mutated BRAF proteins have elevated kinase activity and are transforming in NIH3T3 cells. Furthermore, RAS function is not required for the growth of cancer cell lines with the V599E mutation. As BRAF is a serine/threonine kinase that is commonly activated by somatic point mutation in human cancer, it may provide new therapeutic opportunities in malignant melanoma.

Genomic DNA from 15 cancer cell lines ( 6 breast cancers, 1 smallcell lung cancer (SCLC), 6 non-small-cell lung cancers (NSCLC), 1 mesothelioma, 1 melanoma) and the corresponding matched lym- phoblastoid cell lines from the same individuals were screened for sequence variants through the coding exons and intron-exon junctions of the BRAF gene using a capillary-based modified heteroduplex method followed by direct sequencing of polymerase chain reaction products. (Exon 1, containing 135 base pairs (bp) of coding sequence, failed to amplify despite the use of five different primer sets.) Three single-base substitutions were detected. Two were in BRAF exon 15: T1796A leading to a substitution of valine by glutamic acid at position 599 (V599E) in the melanoma cell line Colo-829, and C1786G leading to L596V in the NSCLC cell line NCI-H2087 (Fig. 1). A further mutation was found in exon 11: G1403C leading to G468A in the NSCLC cell line NCI-H1395. None of the three changes were present in the lymphoblastoid cell lines from the same individuals, indicating that the variants were somatically acquired mutations.

a

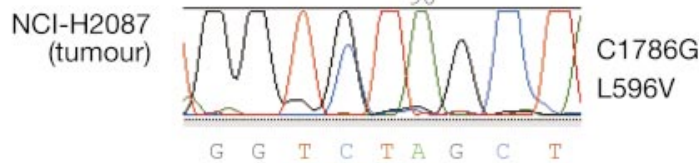

$\mathrm{NCl}-\mathrm{H} 2087$ (normal)
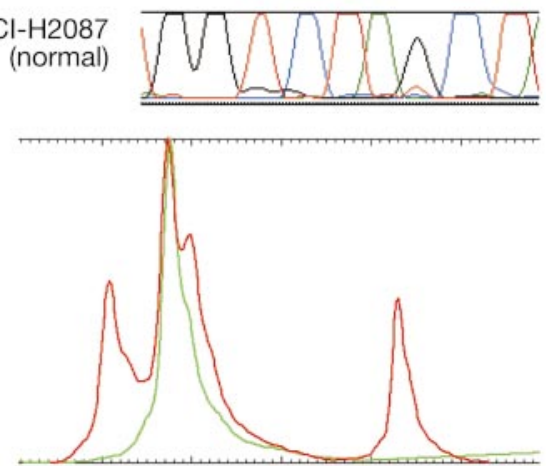

b

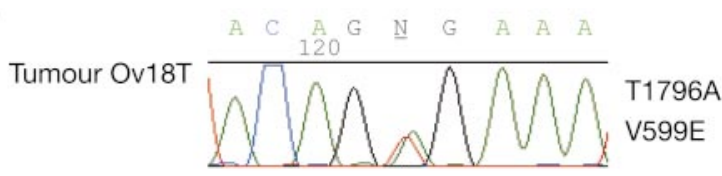

Normal Ov18N
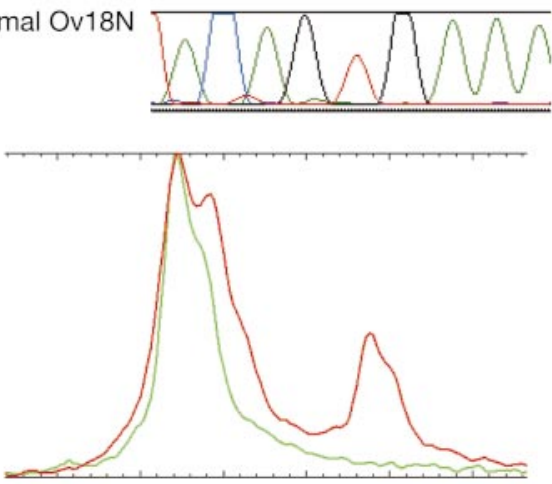

Figure 1 Mutations in the BRAF gene. Sequence electropherograms and corresponding comparisons between heteroduplex traces from normal (green) and cancer (red) DNAs from the same individuals. The heteroduplex trace comparisons are generated using proprietary software (see Methods). For each example (NCl-H2087 cell line (a) and ovarian neoplasm Ov18T (b)) the cancer trace shows additional peaks and/or differently shaped peaks compared with the normal trace. 
To evaluate further the pattern of somatic mutations in $B R A F$, we screened the coding sequence and intron-exon junctions for mutations in genomic DNA from an additional 530 cancer cell lines (Table 1). Presumptive BRAF mutations (excluding variants of unknown significance and germline polymorphisms that were found in 341 normal tissue DNAs) were identified in 43 cancer cell lines including 20 of $34(59 \%)$ melanomas, 7 of $40(18 \%)$ colorectal cancers, 4 of 38 (11\%) gliomas, 4 of 131 (3\%) lung cancers (all four were adenocarcinomas from a total of 35), 5 of 59 (9\%) sarcomas, 1 of $26(4 \%)$ ovarian carcinomas, 1 of $45(2 \%)$ breast cancers and 1 of 7 (14\%) liver cancers. Mutations were not found in cancer cell lines derived from 29 neuroblastomas, 10 bladder cancers, 53 leukaemias and lymphomas, 11 cervical carcinomas, 11 renal cell carcinomas, 3 pancreatic carcinomas, 3 prostate carcinomas, 6 gastric carcinomas, 7 testicular carcinomas, 3 uterine carcinomas and 29 other cancers.

All 43 probable oncogenic BRAF somatic mutations found in the cancer cell lines were in exons 11 and 15 (Table 1). Accordingly, these two exons were screened for mutations in genomic DNA from 378 primary human cancers and short-term cultures (STC, less than passage 15). BRAF mutations were detected in 28 primary cancers/ STCs, including 6 of 9 primary melanomas, 12 of 15 melanoma STCs, 4 of 33 colorectal carcinomas, 5 of 35 ovarian neoplasms (see Fig. 1 for example), and 1 of 182 sarcomas. Mutations were not detected in 33 breast cancers, 15 gliomas, 23 prostate cancers, 14 lung cancers, or 19 head and neck squamous cell carcinomas. Ten of the 35 ovarian tumours examined were classified as borderline (low malignant potential) lesions and 4 of $5 B R A F$ mutations found in ovarian neoplasms were in this subcategory. The single primary sarcoma in which a BRAF mutation was found was classified as a malignant fibrous histiocytoma.

Although BRAF mutations are found in a wide range of cancers, there is a trend towards the occurrence of mutations in cancer types in which a substantial proportion of cases are known to harbour RAS mutations (for example, malignant melanoma, colorectal cancer and borderline ovarian cancers ${ }^{4-6}$ ). The apparent association between the presence of $B R A F$ and RAS mutations in similar cancer types suggests that activation of the RAS-RAF-mitogen-activated protein (MAP)-kinase kinase (MEK)-extracellular signal-regulated kinase (ERK)-MAP kinase pathway can be achieved by mutation at various levels in the pathway and that the pathway is activated in a substantial proportion of cases in these cancer types.

The highest frequency of BRAF mutations is in malignant melanoma (Table 1). This does not seem to be related to the effects of ultraviolet light, the only known environmental risk factor for this disease. The $\mathrm{T} \rightarrow \mathrm{A}$ change at nucleotide 1796, which accounts for 35 of $38(92 \%)$ of BRAF mutations in melanoma (Table 1), is distinct from the $\mathrm{CC} \rightarrow \mathrm{TT}$ or $\mathrm{C} \rightarrow \mathrm{T}$ changes associated with pyrimidine dimer formation following exposure to ultraviolet light-these changes are commonly found, for example, in the TP53 gene in non-melanoma skin cancers ${ }^{7}$.

The high frequency of mutation in melanoma may be related to features of melanocyte biology. $\alpha$-melanocyte stimulating-hormone and other related proopiomelanocortin-derived peptides are crucial regulators in melanocyte biology. $\alpha$-melanocyte stimulating-hormone and proopiomelanocortin-derived peptides bind to the melanocortin receptor I, upregulating cyclic AMP, leading to increased proliferation and melanogenesis in response to UVB radiation $^{8}$. This cAMP-dependent signalling cascade activates BRAF and subsequently ERK ${ }^{9}$. That a principal melanocyte-specific signalling pathway controlling proliferation and differentiation operates through activation of BRAF and that this gene is mutated in most melanomas suggests a possible explanation for the high frequency of $B R A F$ mutation in melanomas relative to other cancer types.

Our analysis reveals mutations in two regions of the BRAF kinase domain. Mutations were very similarly distributed in cancer cell lines and primary cancers. A total of $89 \%$ of mutations are within or immediately adjacent to the activation segment, a region of 10-30 amino acids bounded by almost invariant DFG and APE motifs ${ }^{10}$. Acidic substitutions at a single amino acid residue (usually V599E and one instance of V599D) account for 92\% of activation segment mutations with five further mutations altering residues E585, F594, G595 and L596 (Table 1). These residues are identical at the

\begin{tabular}{|c|c|c|c|c|c|c|c|c|c|c|c|c|c|c|c|c|}
\hline \multicolumn{2}{|l|}{$B R A F$ mutations } & \multicolumn{8}{|c|}{ Cancer cell lines } & \multicolumn{6}{|c|}{ Primary tumours } & \multirow[b]{2}{*}{ Tota } \\
\hline Nucleotide & Amino acid & $\begin{array}{c}(1) \\
\text { Mel. }\end{array}$ & $\begin{array}{c}\text { (2) } \\
\text { Colo. ca. }\end{array}$ & $\begin{array}{c}\text { (3) } \\
\text { Glioma }\end{array}$ & $\begin{array}{c}\text { (4) } \\
\text { Lung ca. }\end{array}$ & $\begin{array}{c}\text { (5) } \\
\text { Sarcoma }\end{array}$ & $\begin{array}{c}\text { (6) } \\
\text { Breast }\end{array}$ & $\begin{array}{c}(7) \\
\text { Ovarian }\end{array}$ & $\begin{array}{c}(8) \\
\text { Other }\end{array}$ & $\begin{array}{c}\text { (1) } \\
\text { Mel. STC }\end{array}$ & $\begin{array}{c}\text { (2) } \\
\text { Mel. }\end{array}$ & $\begin{array}{c}\text { (3) } \\
\text { Colo. ca. }\end{array}$ & $\begin{array}{c}\text { (4) } \\
\text { Ovarian* }^{*}\end{array}$ & $\begin{array}{c}\text { (5) } \\
\text { Sarcoma }\end{array}$ & $\begin{array}{c}(6) \\
\text { Other† }\end{array}$ & \\
\hline $\begin{array}{l}\text { G1388A } \\
\text { G1388T }\end{array}$ & $\begin{array}{l}\text { G463E } \\
\text { G463V }\end{array}$ & & 1 & & & & & 1 & & & & & & & & $\begin{array}{l}1 \\
1\end{array}$ \\
\hline G1394C & G465A & & & & & & & & & 1 & & & & & & 1 \\
\hline G1394A & G465E & & & & & & & & & & 1 & & & & & 1 \\
\hline G1394T & G465V & & & & 1 & & & & & & & & & & & 1 \\
\hline G1403C & G468A & & & & 2 & & & & & & & & & & & 2 \\
\hline G1403A & G468E & & & & & & & & & & & 1 & & & & 1 \\
\hline G1753A & E585K & & & & & & & & & & & & 1 & & & 1 \\
\hline $\mathrm{T} 1782 \mathrm{G}$ & F594L & & & & & & & & & & & 1 & & & & 1 \\
\hline G1783C & G595R & & 1 & & & & & & & & & & & & & 1 \\
\hline C1786G & L596V & & & & 1 & & & & & & & & & & & 1 \\
\hline T1787G & L596R & & & & & & & & & & & & 1 & & & 1 \\
\hline T1796A & V599E & 19 & 5 & 4 & & 5 & 1 & & 1 & 11 & 5 & 2 & 3 & 1 & 0 & 57 \\
\hline TG1796-97AT & V599D & 1 & & & & & & & & & & & & & & 1 \\
\hline & Total & 20 & 7 & 4 & 4 & 5 & 1 & 1 & 1 & 12 & 6 & 4 & 5 & 1 & 0 & 71 \\
\hline No. samples screened & & 34 & 40 & 38 & 131 & 59 & 45 & 26 & 172 & 15 & 9 & 33 & 35 & 182 & 104 & 923 \\
\hline Per cent & & $59 \%$ & $18 \%$ & $11 \%$ & $3 \%$ & $9 \%$ & $2 \%$ & $4 \%$ & $0.6 \%$ & $80 \%$ & $67 \%$ & $12 \%$ & $14 \%$ & $0.5 \%$ & $0 \%$ & $8 \%$ \\
\hline
\end{tabular}

Amino acid residues are grouped in blocks. Three further BRAF coding sequence variants were identified (G2041A R681Q in the HEC1A endometrial cancer cell line, T974C I325T in the ZR-75-30 breast cancer cell line, and C2180T A727V in the H33AJ-JA1 T-ALL cell line). These were not present in 341 control DNAs. Lane numbers (in parentheses) are provided for convenience. Mel., melanoma; Colo. ca., colorectal cancer; Mel. STC, melanoma short-term culture.

${ }^{*}$ Four out of ten LMP (low malignant potential); 1 out of 25 malignant epithelial.

†Glioma $(n=15)$, breast cancer $(n=33)$, prostate cancer $(n=23)$, HNSCC (head and neck squamous cell carcinoma) $(n=19)$, lung cancer $(n=14)$. 
equivalent positions in human RAF1 and $A R A F 1$ and are conserved in all three $R A F$ genes through evolution (with the exception of the BRAF V599 residue in the Drosophila Raf homologue; Fig. 2). A total of $11 \%$ of mutations are in the glycine residues (G463, G465 and G468) of the GXGXXG motif within the glycine-rich loop of the kinase domain. This motif is highly conserved in protein kinases and other proteins that bind mono- and dinucleotides (Fig. 2). The first glycine is present in about $95 \%$ of all kinases, the second in more than $99 \%$ of all kinases and the third in about $85 \%$ of kinases ${ }^{11}$. From structural studies, it has been shown that this region forms a loop that anchors the $\beta$ - and $\gamma$-phosphates of ATP and may orientate ATP for catalysis ${ }^{10}$. Previously described mutants of these glycine residues in other proteins have resulted in reduction of kinase activity ${ }^{12-14}$. In the GTP-binding RAS proteins, however, mutation of G12 within the GXGXXG motif is still compatible with nucleotide binding and results in RAS proteins with transforming activity.

To characterize the biological effects of these mutations, we examined the V599E mutation-because it was the most common-together with one other mutant chosen from the activation segment (L596V) and two mutants of the G loop (G463V, G468A). Myc-epitope-tagged versions of complementary DNAs containing these mutations were transiently expressed in COS cells, immunoprecipitated using the Myc-tag and examined in a kinase cascade assay using bacterially produced glutathione $S$-transferase (GST)MEK, GST-ERK and myelin basic protein (MBP) as sequential substrates $^{15,16}$. All four mutants had elevated basal kinase activity compared with wild-type BRAF ( ${ }^{\mathrm{WT}} \mathrm{BRAF}$, Fig. 3a); however, the basal activity of ${ }^{\mathrm{G} 468 \mathrm{~A}} \mathrm{BRAF}$ and ${ }^{\mathrm{V} 599 \mathrm{E}}$ BRAF was substantially higher (about 12.5- and 10.7-fold that of ${ }^{\mathrm{WT}} \mathrm{BRAF}$, respectively), whereas the activation of ${ }^{\mathrm{G} 463 \mathrm{~V}} \mathrm{BRAF}$ and ${ }^{\mathrm{L} 596 \mathrm{~V}}$ BRAF was more modest (about 2- and 5.7-fold, respectively). All four BRAF proteins also

\section{a}

Human BRAF

Mouse Braf

Chicken Braf

Human RAF1

Mouse Raf1

Xenopus Raf1

Zebrafish Raf1

Drosophila Raf1

Human ARAF1

Mouse Araf

Rat Araf

Pig Araf

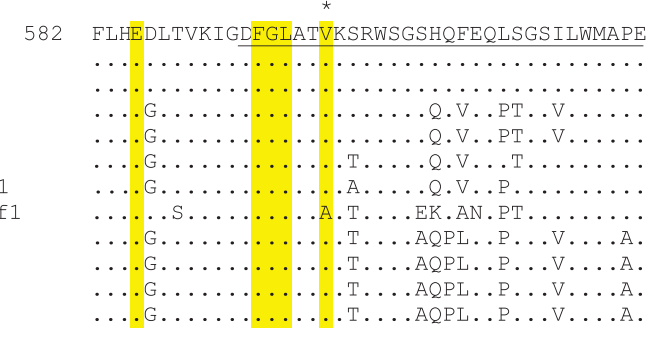

b

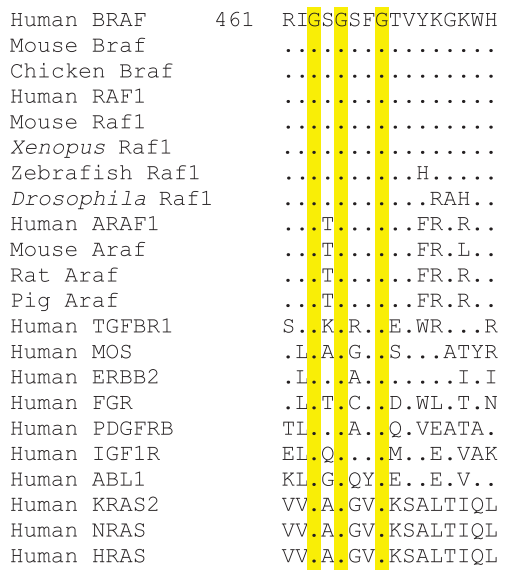

Figure 2 Sequence conservation and mutations in the BRAF activation segment and $\mathrm{G}$ loop. a, b, Conservation of amino acid sequence for the activation segment (a) and G loop (b). The positions of mutations are indicated by yellow shading; V599 is denoted by an asterisk. The activation segment is underlined. stimulated the activity of endogenous ERK when expressed in COS cells as determined by a phospho-specific antibody that only binds to the dually phosphorylated activated form of ERK1/2 (Fig. 3b). Consistent with their in vitro activities, ${ }^{\mathrm{G} 468 \mathrm{~A}} \mathrm{BRAF}$ and ${ }^{\mathrm{V} 599 \mathrm{E}} \mathrm{BRAF}$ stimulated phosphorylation of endogenous ERK1/2 more strongly than ${ }^{\mathrm{G} 463 \mathrm{~V}}$ BRAF or ${ }^{\mathrm{L} 596 \mathrm{~V}}$ BRAF. The data demonstrate that these mutants are active in vitro and stimulate the activity of the pathway in vivo to different degrees. Finally, all four mutants were also stimulated by ${ }^{\mathrm{G} 12 \mathrm{~V}}$ HRAS, although the fold activation for each of the mutants is reduced compared with ${ }^{\mathrm{WT}} \mathrm{BRAF}$ (see Fig. 3a). ${ }^{\mathrm{L} 596 \mathrm{~V}}$ BRAF (about 4.5 -fold stimulation by ${ }^{\mathrm{G} 12 \mathrm{~V}}$ HRAS) was stimulated more strongly than the other mutants tested (between 2- and 2.5-fold stimulation) (Fig. 3a).

The ability of the kinase-activated BRAF mutants to induce transformation was examined by transfection of the epitope-tagged cDNA constructs into NIH3T3 cells to assay focus-forming ability. ${ }^{\mathrm{WT}}$ BRAF transformed cells at very low efficiency $(0.0013$ foci per ng DNA); however, ${ }^{4463 \mathrm{~V}} \mathrm{BRAF},{ }^{\mathrm{G} 468 \mathrm{~A}} \mathrm{BRAF},{ }^{\mathrm{L} 596 \mathrm{~V}} \mathrm{BRAF}$ and ${ }^{\mathrm{V} 599 \mathrm{E}} \mathrm{BRAF}$ transformed NIH3T3 cells $70-138$ times $(0.09-0.18$ foci per ng DNA) more efficiently than ${ }^{\mathrm{WT}} \mathrm{BRAF}$ (Table 2). Inhibiting the kinase activity by substituting alanine for aspartic acid at position 593 within the conserved DFG motif abrogated transform-
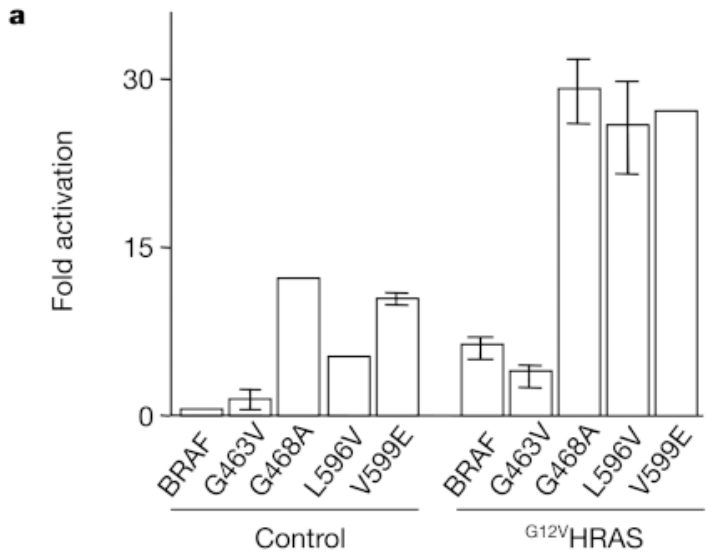

b
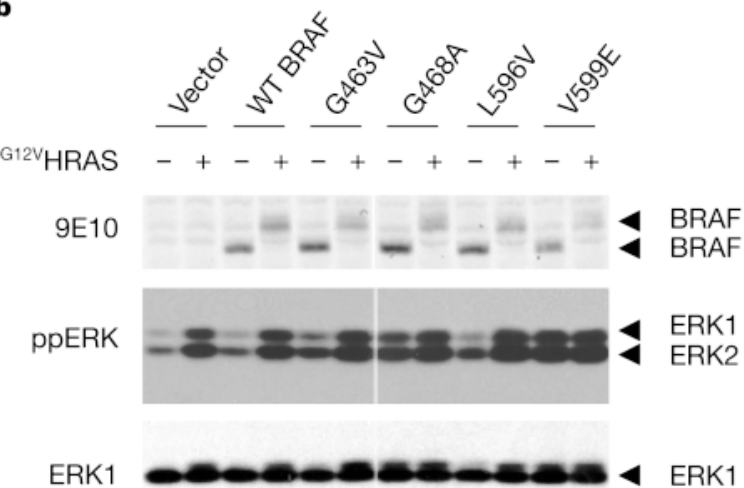

Figure 3 BRAF and ERK activation. a, BRAF kinase activity. Myc-epitope-tagged versions of BRAF and the various mutants were expressed in COS cells alone or in combination with ${ }^{G 12 V}$ HRAS as indicated. The activity of the BRAF proteins in cell extracts was examined using the Raf kinase cascade assay. Each sample was assayed in triplicate and error bars are used to indicate the standard deviations from the mean. Absence of error bars indicates less than $3 \%$ error. Similar results were obtained in two independent transfections. b. ERK phosphorylation. Samples prepared as in a were examined for the presence of phosphorylated, active ERK1/2 (ppERK, middle panel) or total ERK (ERK, bottom panel). Similar results were obtained from extracts from two independent transfection assays. The top panel shows BRAF protein expression in transfectants. 
Table 2 Transforming activity of BRAF mutants

\begin{tabular}{|c|c|c|}
\hline Allele & Transformed foci per $\mu \mathrm{g}$ DNA & Fold increase over wild-type BRA \\
\hline${ }^{w T}$ BRAF & 1.3 & - \\
\hline V599E & 180 & $138 \times$ \\
\hline DAVE & 0 & - \\
\hline L596V & 90 & $70 \times$ \\
\hline DALV & 0 & - \\
\hline G463V & 130 & $100 \times$ \\
\hline G468A & 90 & $69 \times$ \\
\hline $\mathrm{G}_{12 V_{\text {HRAS }}}$ & 12,000 & $9,200 \times$ \\
\hline
\end{tabular}

NIH3T3 cells were transfected as described in Methods. Transformed foci contained cells like Rasor Raf1-transformed cells-which are refractile and frequently bipolar-and often contained the giant cells typical of RAS or RAF1 transformation. DAVE and DALV are kinase-inactive versions of V599E and L596V, respectively, in which D593 of the conserved DFG motif is replaced by alanine to generate a kinase-dead variant.

ing activity, showing that this was dependent on kinase activity. Despite the higher kinase activity of ${ }^{\mathrm{V} 599 \mathrm{E}} \mathrm{BRAF}$ compared with ${ }^{\mathrm{WT}}$ BRAF activated by ${ }^{\mathrm{G} 12 \mathrm{~V}} \mathrm{HRAS},{ }^{\mathrm{V} 599 \mathrm{E}} \mathrm{BRAF}$ has 50 -fold lower transforming activity than ${ }^{\mathrm{G} 12 \mathrm{~V}} \mathrm{HRAS}$ in this assay. This may reflect the fact that whereas RAS signals to a number of effector molecules $^{18}$, RAF proteins may signal predominantly through the ERKMAPK pathway (although other effectors have been proposed ${ }^{2,3,18}$ ). In addition to focus assays, NIH3T3 cells transfected with ${ }^{\mathrm{G} 463 \mathrm{~V}} \mathrm{BRAF}$ and ${ }^{\mathrm{G} 468 \mathrm{~A}} \mathrm{BRAF}$ were assayed for tumorigenicity in nude mice. $10^{6}$ transfected cells pooled from puromycin-resistant colonies produced 5-mm tumours within 18 days (data not shown).

Our results demonstrate that mutations of BRAF found in human cancers activate the kinase. Phosphorylation of residues within the activation segment regulates the activity of many kinases including $\mathrm{BRAF}^{10}$. In BRAF, T598 and S601 within the activation segment both require phosphorylation to achieve maximal kinase activity. These phosphorylations are effected after recruitment of BRAF to the membrane by activated RAS, and replacement of T598 and $\mathrm{S} 601$ by acidic amino acid residues results in RAS-independent activation of $B R A F^{17}$. The V599 mutations found in human cancers probably mimic phosphorylation in the activation segment as they insert a negatively charged residue adjacent to a site of regulatory phosphorylation at S598. Notably, although many studies in experimental systems show that regulatory phosphorylations can be mimicked by substitution of an acidic amino acid, such mutations are probably rare in human disease, because an acidic amino acid substitution cannot be generated from a threonine or serine residue by a single base change. Our data now suggest that amino acids other than threonine and serine can be mutated to acidic residues in human disease to mimic phosphorylation and hence activate kinases. Although the mode of action of V599 mutations may be explicable on the basis of mimicking regulatory phosphorylation, the mechanism of action of the other activation loop mutations is not clear and awaits structural studies. Moreover, the presence in one cancer cell line of an unusual double nucleotide substitution converting V599 to aspartic acid suggests as well that mutations at V599 may be particularly active biologically. Indeed, K600 could be changed by a single base substitution to glutamic acid, yet mutations at this residue have not been found in human cancers.

Our data also demonstrate that mutations of the glycine residues in the GXGXXG motif of the ATP-binding domain can activate kinases. It will be interesting to determine whether mutation of these conserved glycine residues activates other protein kinases and nucleotide-binding proteins. If this proves to be the case then it will provide a useful way to generate constitutively activated kinases experimentally.

The coding exons and intron-exon junctions of HRAS, KRAS and NRAS genes were screened for mutations through the entire panel of 545 cell lines. Seventy-one (13\%) had mutations of RAS genes: 14 of $40(35 \%)$ colorectal cancers, 23 of 131 (18\%) lung cancers (22 NSCLC, 1 SCLC), 3 of 3 (100\%) pancreatic cancers, 3 of 34 (9\%) melanomas, 4 of 26 (15\%) ovarian cancers, 3 of 27 (11\%) neuroblastomas, 2 of 10 (20\%) bladder cancers, 9 of 53 (17\%) leukaemias/ lymphomas and 10 of 221 others. Three of 43 cancer cell lines with $B R A F$ mutations also had RAS gene mutations: $\mathrm{BE}$, a colorectal cancer cell line (KRAS2 G13D; BRAF G463V); Hx62/26, an ovarian cancer cell line (KRAS2 G13D; BRAF G463E); and the NCI-H2087 NSCLC line (NRAS Q61K; BRAF L596V). Mutation screening of the 22 primary cancers with $B R A F$ mutations for which adequate material was available revealed a colorectal cancer with coincident KRAS2 (G12V) and BRAF (F594L) mutations. The four cancers that had coincident RAS and BRAF mutations ( 3 cell lines, 1 primary tumour) were all from the set of 12 screened that had one of the less common BRAF mutations. Conversely, none of the 51 cancer samples with a V599 mutation screened through the RAS genes contained a RAS mutation (see Supplementary Information). This suggests that the common V599E mutation is biologically distinct from the other BRAF mutations.

Studies using injection of the RAS-neutralizing Y13-259 monoclonal antibody have previously shown that most normal cells examined and some tumour cells require RAS function for pro-

\begin{tabular}{|c|c|c|c|c|c|}
\hline Cell line & Tissue & RAS mutation & BRAF mutation & Inhibition of S phase by Y13-259 (\%) & Inhibition of S phase by U0126 (\%) \\
\hline WM-266-4 & Melanoma & WT & V599D & 10 & 99 \\
\hline SK-MEL-28 & Melanoma & WT & V599E & 4 & 98 \\
\hline A2058 & Melanoma & WT & V599E & 0 & 68 \\
\hline Malme & Melanoma & WT & V599E & 0 & ND \\
\hline Colo741 & Colorectal & WT & V599E & 0 & 76 \\
\hline LS411N & Colorectal & WT & V599E & 0 & 35 \\
\hline НT29 & Colorectal & WT & V599E & 15 & ND \\
\hline Colo205 & Colorectal & WT & V599E & 3 & ND \\
\hline Mawi & Colorectal & WT & V599E & 5 & 8 \\
\hline $\mathrm{NCl}-\mathrm{H} 1666$ & NSCLC & WT & G465V & 89 & ND \\
\hline $\mathrm{BE}$ & Colorectal & G13DKRAS2 & G463V & 97 & ND \\
\hline NCl-H2087 & NSCLC & ${ }^{\mathrm{Q} 61 \mathrm{~K}_{\mathrm{NRAS}}}$ & L596V & 77 & 56 \\
\hline Lim1899 & Colorectal & ${ }^{G 12 A}$ KRAS2 & WT & 74 & ND \\
\hline LS174T & Colorectal & G12DKRAS2 & WT & 84 & ND \\
\hline JW2 & Colorectal & G12DKRAS2 & WT & 79 & ND \\
\hline SW620 & Colorectal & G12V KRAS2 & WT & 92 & 92 \\
\hline DLD1 & Colorectal & G13DKRAS2 & WT & 4 & ND \\
\hline HCT-116 & Colorectal & G13DKRAS2 & WT & 95 & 75 \\
\hline SK-MEL-2 & Melanoma & Q61RNRAS & WT & 7 & 62 \\
\hline HMVII & Melanoma & ${ }^{\mathrm{Q} 61 \mathrm{~K}}$ NRAS & WT & 100 & 86 \\
\hline $\mathrm{CHL}$ & Melanoma & WT & WT & 96 & 51 \\
\hline SK-MEL-31 & Melanoma & WT & WT & 22 & 98 \\
\hline
\end{tabular}

ND, not done; WT, wild type; NSCLC, non-small-cell lung cancers. 
liferation in culture ${ }^{19,20}$. Microinjection of Y13-259 into eight cell lines with the V599E mutation and the cell line with V599D did not block their proliferation in culture (Table 3 ). However, three cell lines tested with less common BRAF mutations (two of which had RAS mutations and one of which did not, Table 3 ) were inhibited by Y13-259 microinjection. These data suggest that BRAF V599 mutations uncouple cells from their proliferation requirement of RAS (although our in vitro data indicate that BRAF V599 mutants can be even further activated by mutant RAS), whereas other BRAF mutants remain dependent on RAS function. One interpretation of these results is that the less common $B R A F$ mutants still require interaction with RAS to become phosphorylated and activated, whereas V599 mutants overcome the need for a RAS-dependent step by mimicking phosphorylation. The coincidence of $R A S$ and $B R A F$ mutants in the same cancer cell is, to our knowledge, the first report of tandem-activating mutations in more than one component of this signalling pathway. The observation prompts the speculation that mutant RAS signalling may be modulated by mutations at other locations in the pathway.

RAF proteins phosphorylate MEK1/2, which in turn phosphorylate ERK1/2. To evaluate whether activating mutations of $B R A F$ signal through MEK and ERK, we treated cells with the MEK1/2 inhibitor U0126 (ref. 21). This compound inhibits DNA synthesis in a wide variety of cell types ${ }^{21}$. Of those cancer cell lines in which treatment with U0126 blocked ERK1/2 phosphorylation by at least $80 \%, 6$ of 6 with either V599E or V599D mutations showed strong inhibition of DNA synthesis (Table 3). These results are therefore consistent with the hypothesis that the activated versions of BRAF signal, at least in part, through the classical MAPK cascade to promote proliferation.

Examination of the exons and splice junctions of RAF1 in the set of 545 cancer cell lines did not reveal evidence of frequent mutations in melanoma or any other cancer. $B R A F$ may be preferred as a mutational target because it has a higher basal kinase activity than $R A F 1$ (ref. 15). Indeed, when we introduced the BRAF mutations found in human cancers into the cognate positions in $R A F 1$, they had at least 10-fold lower activity in kinase and transformation assays (data not shown). This may be due to the fact that RAF1 requires phosphorylation on serine 338 and tyrosine 341 for activation of the kinase $^{15}$. By contrast, in BRAF, the equivalent serine (S445) is constitutively phosphorylated and the position equivalent to the tyrosine is substituted by an aspartic acid residue (D448), which acts as a phosphomimetic. Thus BRAF may require fewer post-translational modifications than RAF1 to achieve maximal kinase activity and hence is more susceptible to oncogenic activation.

We have identified BRAF as an oncogene in human cancer. The pattern and activity of mutations observed is likely to yield new insights into kinase function. There has recently been marked success reported of an inhibitor (STI571) of the BCR-ABL kinase in the treatment of chronic myeloid leukaemia ${ }^{22}$. The high frequency of $B R A F$ mutations in melanoma and the relative lack of effective therapies for advanced stages of this disease suggest that inhibition of BRAF activity may be an important new strategy in the treatment of metastatic melanoma. The identification of $B R A F$ as a commonly mutated target in human cancer at such an early stage of our genome-wide screen suggests that systematic searches through cancer cell genomes for somatic mutations ultimately will provide a much more complete picture of the number and patterns of mutations underlying human oncogenesis.

\section{Methods}

\section{Tissue samples}

Normal and neoplastic tissue samples were obtained from publicly available banks in the case of cell lines, and from individual investigators using appropriate local Institutional Review Board approved protocols/ethical approval procedures for tissue collection in the case of primary tumour material. Cord blood controls were obtained from the North Cumbria Community Genetics Project (NCCGP). All 545 cancer cell lines and the 15 normal lymphoblastoid lines were genotyped using 400 polymorphic microsatellites (ABI linkage MD-10 panel) to confirm both matching of normal and tumour cell lines as well as non-duplication of cell lines.

\section{Mutation screening}

Screening for mutations was performed using a capillary-based modified heteroduplex method optimized to run on an ABI PRISM 3100 Genetic Analyser ${ }^{23}$ (H.D., manuscript in preparation). PCR primers were designed to amplify the exon plus at least $50 \mathrm{bp}$ of flanking intronic sequence (see Supplementary Information for primer sequences). A total of $12 \mathrm{ng}$ genomic DNA from the test sample was mixed with $3 \mathrm{ng}$ control genomic DNA and amplified using standard PCR conditions in which one of the primers was labelled with either FAM, NED or VIC dye. The resulting samples were then analysed on an ABI PRISM 3100 Genetic Analyser under semi-denaturing conditions using optimized separation medium and run conditions. The resulting traces were analysed using proprietary software to identify samples that produced a shift in peak migration relative to either the matched normal control from the same individual or a standard normal control, indicating the presence of a putative sequence variation. Samples that produced a heteroduplex shift were directly sequenced on both strands using BigDye terminator Cycle Sequencing Ready Reaction Kit (Applied Biosystems) according to the manufacturer's protocol, and analysed on an ABI PRISM 3100 Genetic Analyser.

\section{RAS-neutralizing antibody microinjection and U0126 treatment}

Tumour cells were seeded at a density such that they were approximately $50 \%$ confluent on the day of injection. Cells were microinjected with Y13-259 or control immunoglobulin- $\gamma$ as described previously ${ }^{24}$. Twenty hours after microinjection cells were labelled with 5bromodeoxyuridine (BrdU) for $24 \mathrm{~h}$, fixed and stained with antibody against BrdU. We scored at least 100 microinjected cells for each experiment. To determine the effects of inhibiting ERK1/2 activation on proliferation of tumour cells in culture, cells were seeded on day 1 then the following day U0126 was added in DMSO to $10 \mu \mathrm{M}$; after $20 \mathrm{~h} \mathrm{BrdU}$ was added for a further $24 \mathrm{~h}$ before fixation and staining with antibody against BrdU. Lysates were also made from cells treated in parallel to study the level of inhibition of ERK1/2 phosphorylation by western blotting with an antibody specific for the di-phosphorylated activated forms of ERK1/2 (clone MAP-YT; Sigma)

\section{Transformation assays}

$1.3 \times 10^{5}$ cells of clone D4 of NIH 3T3 cells were transfected with 15-450 ng BRAF expression plasmids in pEFm6 (ref. 17) together with $50 \mathrm{ng}$ pBabe Puro and sufficient empty pEFm6 to give a total of 700 ng DNA, using Lipofectamine (Invitrogen). After 24$26 \mathrm{~h}$, cells were trypsinized and divided between two $10-\mathrm{cm}$ tissue culture dishes containing DMEM plus 5\% donor calf serum (focus assay) and two $10-\mathrm{cm}$ dishes containing $2.5 \mu \mathrm{g} \mathrm{ml}^{-1}$ puromycin in DMEM plus 5\% donor calf serum (transformed colony assay). The medium of the focus assays was changed every four days whereas the medium of the transformed colony assay was changed after seven days. Plates were scored, by experimenters 'blind' for transformation, after 12-18 days. $10^{6}$ cells from pooled colonies of cells from the puromycin-selected dishes were injected subcutaneously into male nude mice aged over six weeks. Mice were observed twice weekly and killed when tumours reached a size in excess of $5 \times 5 \mathrm{~mm}$.

\section{BRAF activity assays}

Myc-epitope-tagged versions of BRAF were transiently expressed in COS cells using the reagent lipofectamine (Gibco/BRL) according to the manufacturer's instructions. Cell extracts were prepared and the Myc-epitope-tagged protein kinase activity was determined as described previously ${ }^{15,16}$. Western blotting for ERK and ppERK was performed as described ${ }^{15,16}$.

Received 2 February; accepted 16 May 2002; doi:10.1038/nature00766.

Published online 9 June 2002.

Peyssonnaux, C. \& Eychène, A. The Raf/MEK/ERK pathway: new concepts of activation. Biol. Cell 93, 53-62 (2001).

2. Avruch, J. A. et al. Ras activation of the Raf kinase: tyrosine kinase recruitment of the MAP kinase cascade. Recent Prog. Horm. Res. 56, 127-155 (2001)

3. Kolch, W. Meaningful relationships: the regulation of the Ras/Raf/MEK/ERK pathway by protein interactions. Biochem. J. 351, 289-305 (2000)

4. Vogelstein, B. et al. Genetic alterations during colorectal-tumour development. N. Engl. J. Med. 319, 525-532 (1988).

5. van't Veer, L. J. et al. N-ras mutations in human cutaneous melanoma from sun-exposed body sites. Mol. Cell Biol. 9, 3114-3116 (1989).

6. Caduff, R. F., Svoboda-Newman, S. M., Ferguson, A. W., Johnston, C. M. \& Frank, T. S. Comparison of mutations of Ki-RAS and p53 immunoreactivity in borderline and malignant epithelial ovarian tumours. Am. J. Surg. Pathol. 23, 323-328 (1999).

7. Daya-Grosjean, L., Dumaz, N. \& Sarasin, A. The specificity of p53 mutation spectra in sunlight induced human cancers. J. Photochem. Photobiol. B 28, 115-124 (1995).

8. Halaban, R. The regulation of normal melancyte proliferation. Pigment Cell Res. 13, 4-14 (2000).

9. Busca, R. et al. Ras mediates the cAMP-dependent activation of extracellular signal-regulated kinases (ERKs) in melanocytes. EMBO J. 19, 2900-2910 (2000).

10. Johnson, L. N., Lowe, E. D., Noble, M. E. \& Owen, D. J. The Eleventh Datta Lecture. The structural basis for substrate recognition and control by protein kinases. FEBS Lett. 430, 1-11 (1998).

11. Hemmer, W., McGlone, M., Tsigelny, I. \& Taylor, S. S. Role of the glycine triad in the ATP-binding site of cAMP-dependent protein kinase. J. Biol. Chem. 272, 16946-16954 (1997).

12. Grant, B. D., Hemmer, W., Tsigelny, I., Adams, J. A. \& Taylor, S. S. Kinetic analyses of mutations in the glycine-rich loop of cAMP-dependent protein kinase. Biochemistry 37, 7708-7715 (1998).

13. Odawara, M. et al. Human diabetes associated with a mutation in the tyrosine kinase domain of the insulin receptor. Science 245, 66-68 (1989). 
14. Cooke, M. P. \& Perlmutter, R. M. Expression of a novel form of the fyn proto-oncogene in hematopoietic cells. New Biol. 1, 66-74 (1989)

15. Mason, C. S. et al. Serine and tyrosine phosphorylations cooperate in Raf-1, but not B-Raf activation. EMBO J. 18, 2137-2148 (1999).

16. Marais, R., Light, Y., Paterson, H. F., Mason, C. S. \& Marshall, C. J. Differential regulation of Raf-1, ARaf, and B-Raf by oncogenic ras and tyrosine kinases. J. Biol. Chem. 272, 4378-4383 (1997).

17. Zhang, B. H. \& Guan, K. L. Activation of B-Raf kinase requires phosphorylation of the conserved residues Thr598 and Ser601. EMBO J. 19, 5429-5439 (2000)

18. Vojtek, A. B. \& Der, C. J. Increasing complexity of the Ras signalling pathway. J. Biol. Chem. 273, 19925-19928 (1998)

19. Mulcahy, L. S., Smith, M. R. \& Stacey, D. W. Requirement for ras proto-oncogene function during serum-stimulated growth of NIH 3T3 cells. Nature 313, 241-243 (1985).

20. Stacey, D. W., DeGudicibus, S. R. \& Smith, M. R. Cellular ras activity and tumour cell proliferation Exp. Cell Res. 171, 232-242 (1987)

21. Favata, M. F. et al. Identification of a novel inhibitor of mitogen-activated protein kinase kinase. J. Biol. Chem. 273, 18623-18632 (1998).

22. Druker, B. J. et al. Efficacy and safety of a specific inhibitor of the BCR-ABL tyrosine kinase in chronic myeloid leukaemia. N. Engl. J. Med. 344, 1031-1037 (2001).

23. Rozycka, M., Collin, N., Stratton, M. R. \& Wooster, R. Rapid detection of DNA sequence variants by conformation sensitive capillary electrophoresis. Genomics 70, 34-40 (2000).

24. Mittnacht, S., Paterson, H., Olson, M. F. \& Marshall, C. J. Ras signalling is required for inactivation of the tumour suppressor pRb cell-cycle control protein. Curr. Biol. 7, 219-221 (1997).

Supplementary Information accompanies the paper on Nature's website

(http://www.nature.com/nature)

\section{Acknowledgements}

We would like to thank all the patients who donated samples for these studies, the UK Children's Cancer Study Group for provision of paediatric primary tumour samples, the NCCGP for provision of cord blood control DNA samples, and W. Haynes for assistance with preparation of the manuscript. We would also like to acknowledge the Wellcome Trust, Institute of Cancer Research and Cancer Research UK for support. C.J.M. is a Gibb life fellow of the Cancer Research UK. G.P. and A.C. are funded in part by Regione Autonoma della Sardegna. B.A.G. is supported by Breakthrough Breast Cancer.

\section{Competing interests statement}

The authors declare that they have no competing financial interests.

Correspondence and requests for materials should be addressed to R.W. (e-mail: rw1@sanger.ac.uk).

\section{VEGF regulates haematopoietic stem cell survival by an internal autocrine loop mechanism}

Hans-Peter Gerber*, Ajay K. Malik, Gregg P. Solar†, Daniel Sherman*, Xiao Huan Liang*, Gloria Meng $\neq$, Kyu Hong $\neq$, James C. Marsters $\$ \& Napoleone Ferrara*

Departments of ${ }^{\star}$ Molecular Oncology, $\dagger$ Protein Chemistry, $\neq$ Assay Development and $\$$ Organic Chemistry, Genentech, Inc., 1 DNA Way, South San Francisco, California 94080, USA

Vascular endothelial growth factor (VEGF) is a principal regulator of blood vessel formation and haematopoiesis ${ }^{1,2}$, but the mechanisms by which VEGF differentially regulates these processes have been elusive. Here we describe a regulatory loop by which VEGF controls survival of haematopoietic stem cells (HSCs). We observed a reduction in survival, colony formation and in vivo repopulation rates of HSCs after ablation of the VEGF gene in mice. Intracellularly acting small-molecule inhibitors of VEGF receptor (VEGFR) tyrosine kinase dramatically reduced colony formation of HSCs, thus mimicking deletion of the VEGF gene. However, blocking VEGF by administering a soluble VEGFR-1, which acts extracellularly, induced only minor effects. These findings support the involvement in HSC survival of a VEGF-dependent internal autocrine loop mechanism (that is, the mechanism is resistant to inhibitors that fail to penetrate the intracellular compartment). Not only ligands selective for VEGF and VEGFR-2 but also VEGFR-1 agonists rescued survival and repopulation of VEGF-deficient HSCs, revealing a function for VEGFR-1 signalling during haematopoiesis.

Differentiation, maintenance and expansion of HSCs is part of a highly orchestrated process involving multiple growth factors, cytokines and chemokines, which act in complex circuits of paracrine and autocrine regulation. Stromal cells such as fibroblasts, macrophages, $\mathrm{T}$ lymphocytes and endothelial cells residing in the bone marrow secrete a range of cytokines regulating the maintenance of HSCs as well as their rapid expansion during pathologic conditions. The apparent physical association of HSCs with stromal cells at sites of HSC maintenance and differentiation suggested paracrine and juxtacrine (membrane-anchored) mechanisms. However, various growth factors and cytokines including interleukin (IL)-3, granulocyte-macrophage colony-stimulating factor (GM-CSF), IL-6 and VEGF are co-expressed with their respective receptors on normal, early and differentiated haematopoietic cells, suggesting that autocrine mechanisms are involved in the regulation of haematopoiesis (for review see ref. 3 ).

VEGF is expressed in bone marrow, and VEGF levels in HSCs increase in response to cytokine stimulation ${ }^{4}$. VEGFR-2 is present

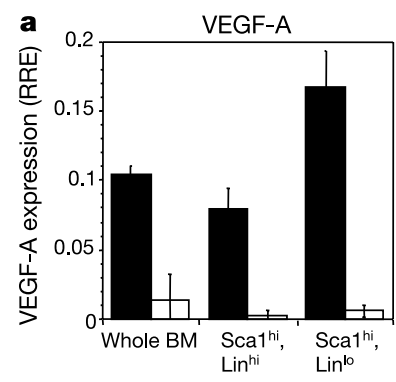

- VEGF/ox/lox $\square$ VEGFflox/flox
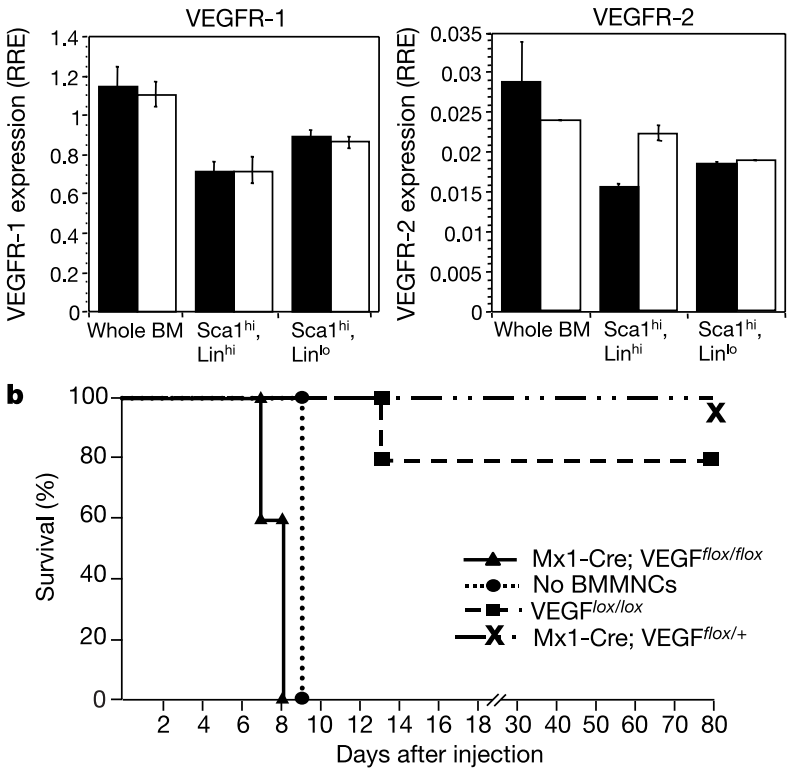

Figure 1 Real-time RT-PCR analysis of BMMNCs and HSCs and competitive repopulation frequencies of VEGF-deficient HSCS. a, VEGF ${ }^{\text {floxflox }}$ cells are derived from VEGF ${ }^{\text {lox/lox }}$ cells following gene ablation. Total RNA was isolated from $1 \times 10^{6} \mathrm{VEGF}^{\text {flox }}$ flox and VEGF ${ }^{\text {lox } / \text { lox }}$ BMMNCs or HPCs $\left(\mathrm{Sca}^{+}\right)$that were lineage committed $\left(\mathrm{Lin}^{\text {hi }}\right)$ or not $\left(\mathrm{Lin}^{10}\right) 4 \mathrm{~d}$ after addition of IFN- $\alpha$. Relative RNA units (RRE) for VEGFR-1 (FIt-1), VEGFR-2 (KDR/FIk-1) and VEGF-A were normalized to glyceraldehyde-3-phosphate dehydrogenase (GAPDH) levels and calculated from standard curves ${ }^{28}$. Data shown are means \pm s.d. from three RNA preparations. BM, bone marrow. b. Survival experiment of lethally irradiated C57BL/6 mice (five mice per group) repopulated with wild-type (VEGF ${ }^{\text {lox/lox }}$ ), heterozygous $\left(\mathrm{Mx1}\right.$-Cre; VEGF $\left.{ }^{f l o x}++\right)$ and VEGF-deficient (Mx1-Cre; VEGF ${ }^{\text {floxflox })}$ BMMNCs. 